\title{
Frailty and health care use among community- dwelling older adults with diabetes: a population- based study
}

This article was published in the following Dove Press journal:

Clinical Interventions in Aging

\author{
Chia-Lin $\mathrm{Li}^{1,2}$ \\ Fiona F Stanaway ${ }^{3}$ \\ Jen-Der Lin ${ }^{2}$ \\ Hsing-Yi Chang ${ }^{4}$ \\ 'Department of Health Care \\ Management, College of Management, \\ Chang Gung University, Kwei-Shan, \\ Tao-Yuan 333, Taiwan; ${ }^{2}$ Division of \\ Endocrinology and Metabolism, \\ Department of Internal Medicine, \\ Chang Gung Memorial Hospital, \\ Chang Gung University, Linkou, \\ Tao-Yuan 333, Taiwan; ${ }^{3}$ Sydney School \\ of Public Health, University of \\ Sydney, Sydney 2006, NSW, Australia; \\ ${ }^{4}$ Division of Preventive Medicine and \\ Health Service Research, Institute of \\ Population Health Sciences, National \\ Health Research Institutes, Maoli 350, \\ Taiwan
}

Purpose: The aims of this study were to investigate the prevalence of frailty and its relationship with health care use among community-dwelling older adults with diabetes.

Methods: We analyzed data from a nationally representative sample of people aged 65 years and above $(n=3,203)$ participating in the 2013 National Health Interview Survey in Taiwan. A total of 719 participants had a history of self-reported physician-diagnosed diabetes. The presence of frailty was determined based on the Fatigue, Resistance, Ambulation, Illness, and Loss of weight (FRAIL) scale proposed by the International Association of Nutrition and Aging. FRAIL scores range from 0 to 5 and are categorized as frail (3-5), pre-frail (1-2), and robust (0). Participants were asked whether they had been hospitalized or had visited an emergency department in the past year.

Results: Among community-dwelling older adults with diabetes, $9.4 \%$ of participants were frail and $35.3 \%$ were pre-frail. After adjustment for other factors, being frail was significantly associated with hospitalization during the past year $(\mathrm{OR}=5.31,95 \% \mathrm{CI}=1.87-15.10)$, whereas being pre-frail was not associated with hospitalization. Both being pre-frail and frail were significantly associated with emergency department visits during the past year $(\mathrm{OR}=2.64,95 \% \mathrm{CI}=1.35-5.17$ and $\mathrm{OR}=4.05,95 \% \mathrm{CI}=1.31-12.49$, respectively) after adjustment for other factors.

Conclusion: Our results highlight the high prevalence of frailty in community-dwelling older adults with diabetes. Furthermore, being frail is associated with a greater burden of hospitalizations and emergency department visits.

Keywords: diabetes mellitus, elderly, frailty, health care use, Taiwan

\section{Introduction}

Despite the varying definitions of frailty used in the literature, studies have consistently demonstrated a high prevalence of frailty in older adults with diabetes. ${ }^{1-5}$ Frailty is characterized by deterioration in multiple organ systems, along with declining cardiopulmonary reserve and loss of executive function. ${ }^{6}$ Frailty is associated with higher mortality risk among middle-aged and older adults with diabetes. ${ }^{7,8}$ These findings highlight the importance of early identification of frailty for health care planning and preventing poor outcomes in older people with diabetes. In Taiwan, $\sim 25 \%$ of adults aged 65 years and older have diabetes, ${ }^{9}$ and diabetes has been the fourth or fifth leading cause of death since 2002. However, to date the frailty profile and associated health care use among community-dwelling older adults with diabetes in this population have not yet been adequately characterized.

Liccini and Malmstrom used the Fatigue, Resistance, Ambulation, Illness, and Loss of weight (FRAIL) scale proposed by the International Academy on Nutrition and Aging 
in an observational study of 198 outpatients with diabetes aged 50-90 years. They found that $28.8 \%$ of participants with diabetes were classified as frail and that these participants had a higher likelihood of hospitalization over a 6-month period than non-frail participants with diabetes. ${ }^{10} \mathrm{Li}$ et al also used the FRAIL scale in a pilot study of 146 inpatients aged 60 years or older with type 2 diabetes and found that $15.1 \%$ of participants were classified as frail at hospital admission, and that $40.9 \%$ of those with frailty had three or more hospitalizations within 1 year after discharge. ${ }^{11}$ This highlights that being frail is common and often under-recognized in older people with diabetes. However, both these studies have been conducted on clinical populations, and there is a lack of studies investigating health care use in frail, pre-frail, and non-frail participants in a population-based sample of community-dwelling older adults with diabetes. Understanding the relationship between frailty and health care use among community-dwelling older adults with diabetes has important implications for resource allocation and implementation of strategies for prevention and management of frailty in this population.

In view of these considerations, we analyzed data from a national sample of older adults aged 65 years or older who participated in the 2013 National Health Interview Survey (NHIS) in Taiwan. The aims of the present study were twofold. First, we investigated the prevalence of frailty among community-dwelling older adults with diabetes. Second, we investigated the rates of all-cause hospital admission and emergency department visits during the past year among community-dwelling older adults with diabetes by frailty status. We hypothesized that among older adults with diabetes, those with frailty would have greater odds of hospital admission and emergency department visits compared to robust participants.

\section{Methods}

\section{Study population}

The present study includes participants from the 2013 NHIS in Taiwan. This survey used a complex multistage stratified systematic sampling design that is described in detail on the NHIS website (http://nhis.nhri.org.tw/) and in a previous publication. ${ }^{12}$ The survey obtained ethical approval from the Institutional Review Board of the National Health Research Institutes. All study participants provided signed written informed consent. The original sample comprised 23,296 participants (response rate 75.2\%), including 3,203 individuals aged 65 years and older. Of these, a total of 719 individuals with a self-reported physician-confirmed diagnosis of diabetes were eligible for analysis in this study.

\section{Assessment of frailty}

In this study, frailty was measured using the FRAIL scale proposed by the International Academy on Nutrition and Aging. ${ }^{13,14}$ FRAIL includes five criteria: fatigue, resistance, ambulation, illnesses, and loss of weight. Fatigue was assessed by asking how much of the time participants felt tired in the past 1 week. Responses of "all" or "most of the time" were given a score of 1 . Resistance was assessed by asking participants whether they have difficulty climbing ten steps, and ambulation was assessed by asking participants whether they have difficulty walking $400 \mathrm{~m}$. Responses of "some difficulty", "much difficulty", or "unable to carry out" received a score of 1 . Illnesses were assessed by asking participants if a medical professional had ever told them that they had any of the following illnesses: hypertension, cancer, diabetes, chronic lung disease, heart disease, asthma, arthritis, stroke, and kidney disease. A report of five or more illnesses received a score of 1 . In the FRAIL scale, weight loss of more than $5 \%$ scores 1 point. However, there was no equivalent variable available from the 2013 NHIS database. Thus, in this study, loss of weight was assessed by body mass index (BMI was calculated as weight $[\mathrm{kg}]$ divided by height squared $\left[\mathrm{m}^{2}\right]$ ), and participants scored 1 point if their BMI was less than $18.5 \mathrm{~kg} / \mathrm{m}^{2} .{ }^{15}$ FRAIL scores ranged from 0 to 5. Participants with scores ranging from 3 to 5 were defined as frail, 1-2 as pre-frail, and 0 as robust. The FRAIL scale has been validated in previous studies. ${ }^{15}$ In Hong Kong, Woo et al found that the FRAIL scale was comparable to other existing screening tools in predicting mortality and physical limitation in the Chinese population. ${ }^{15}$

\section{Demographic characteristics and assessment of falls, activities of daily living (ADL), and instrumental activities of daily living (IADL) disability}

Basic demographic information such as age, sex, years of education, and marital status was obtained from the questionnaires. Participants with diabetes were also asked about their age at diagnosis and the use of insulin. Participants were categorized as having fallen if they self-reported at least one fall during the previous year. Participants were asked to report their ability to perform six ADL including eating, bathing, dressing, using the toilet, getting in or out of bed, and walking across a small room, and five IADL including preparing meals, grocery shopping, using the telephone, taking medications, and managing money. Participants were asked whether they could carry out these ADL and IADL activities with no difficulty, some difficulty, much difficulty, 
or were unable to carry them out. Limitation in each task was dichotomized as no difficulty or some difficulty vs much difficulty or complete inability. Disability for each scale was defined as reporting limitations in one or more tasks in the respective scale.

\section{Assessment of health care use}

The dependent variables were respondents' self-reported hospital admission or emergency department visit in the previous year before the interview. Hospital admissions and emergency department visits were assessed as dichotomous variables (any or none).

\section{Statistical analysis}

We used the Pearson's chi-squared test to examine factors associated with frailty status among older adults with diabetes. Multiple logistic regression analysis was used to examine the association of frailty status and other factors with hospital admission and emergency department visits and for estimation of ORs and 95\% CIs. To account for the complex sampling design, all analyses were carried out using SAS (SAS Institute Inc., Cary, NC, USA)-callable SUDAAN (RTI, Research Triangle Park, NC, USA). Sampling weights were applied to make the sample representative of the whole Taiwanese population.

\section{Results}

Table 1 presents the characteristics of older adults with diabetes by frailty status. Among older adults with diabetes, $9.4 \%$ were categorized as frail and $35.3 \%$ as pre-frail.
Older adults with diabetes who were also frail were more likely to be older, unmarried, live alone, have a lower level of education, have a longer duration of diabetes, be using insulin, have fallen during the last year, and report ADL and IADL disability.

Table 2 presents the rates and OR and 95\% CI for participants with diabetes for hospitalization and emergency department visits during the past year. After adjustment for other factors, compared to participants with diabetes who were robust, participants with diabetes who were pre-frail had an OR for hospital admission during the past year of $1.90(95 \%$ CI $=0.91-3.96)$ and had an OR for emergency department visits of 2.64 (95\% CI $=1.35-5.17)$. In addition, compared to robust participants those with diabetes who were frail had an OR for hospital admission during the past year of $5.31(95 \% \mathrm{CI}=1.87-15.10)$ and had an OR for emergency department visits of 4.05 (95\% CI $=1.31-12.49)$.

\section{Discussion}

Our results confirm that there is high prevalence of frailty among community-dwelling older adults with diabetes. In addition, in older adults with diabetes, the rates of hospital admission during the last year for participants who were frail $(55.0 \%)$ or pre-frail $(33.1 \%)$ were significantly higher than those of robust participants (15.4\%). Similarly, the proportion of participants who had at least one emergency department visit during the past year was $45.2 \%$ among frail individuals, $36.2 \%$ among pre-frail individuals, and $16.8 \%$ among robust participants. Among older adults with diabetes, after adjustment for other factors, frailty was associated with a greater

Table I Characteristics of participants by frailty status

\begin{tabular}{|c|c|c|c|c|c|}
\hline \multirow[t]{2}{*}{ Characteristics } & \multirow[t]{2}{*}{ Total } & \multicolumn{3}{|l|}{ Frail } & \multirow[t]{2}{*}{$P$-value ${ }^{a}$} \\
\hline & & Robust & Pre-frail & Frail & \\
\hline N (\%) & $719(100)$ & $383(55.4)$ & $270(35.3)$ & $66(9.4)$ & \\
\hline Age, years (\%) & & & & & $<0.0001$ \\
\hline $65-74$ & 58.4 & 71.0 & 43.4 & 40.2 & \\
\hline $75+$ & 41.6 & 29.0 & 56.6 & 59.8 & \\
\hline Sex (\% female) & 58.0 & 53.7 & 61.8 & 69.6 & 0.0948 \\
\hline Education (\%) & & & & & 0.0141 \\
\hline 0 years & 29.5 & 22.9 & 35.1 & 47.9 & \\
\hline $\mathrm{I}-6$ years & 44.1 & 45.9 & 42.0 & 41.7 & \\
\hline $7+$ years & 26.4 & 31.2 & 23.0 & 10.5 & \\
\hline Marital status (\% married or living with partner) & 63.8 & 70.5 & 56.5 & 51.2 & 0.0215 \\
\hline Duration of diabetes ( $\geq 10$ years, $\%$ ) & 45.1 & 37.6 & 58.0 & 45.3 & 0.0084 \\
\hline Use of insulin (\% yes) & 12.1 & 6.3 & 15.2 & 33.3 & 0.0040 \\
\hline Fallen during past year (\% yes) & 18.9 & 12.8 & 24.5 & 33.7 & 0.0111 \\
\hline ADL disability (\% yes) & 15.4 & 0 & 28.8 & 56.3 & $<0.0001$ \\
\hline IADL disability (\% yes) & 27.6 & 5.3 & 49.9 & 75.6 & $<0.0001$ \\
\hline
\end{tabular}

Note: a Categorical variables were compared using Pearson's chi-squared test and shown as percentages.

Abbreviations: ADL, activities of daily living; IADL, instrumental activities of daily living. 
Table 2 Rates, ORs, and 95\% Cls for hospitalization and emergency department visits during the past year

\begin{tabular}{|c|c|c|c|c|c|}
\hline & \multirow[t]{2}{*}{ Robust ( $\mathbf{N}=383$ ) } & \multicolumn{2}{|c|}{ Pre-frail $(\mathrm{N}=270)$} & \multicolumn{2}{|l|}{ Frail $(\mathrm{N}=66)$} \\
\hline & & OR $(95 \% \mathrm{Cl})$ & $P$-value & OR $(95 \% \mathrm{Cl})$ & $P$-value \\
\hline \multicolumn{6}{|l|}{ Hospitalization } \\
\hline Rate (\%) & 15.4 & 33.1 & & 55.0 & \\
\hline Unadjusted OR & Reference & $2.72(1.55-4.77)$ & 0.0011 & 6.71 (2.92-15.38) & 0.0001 \\
\hline Adjusted $\mathrm{OR}^{\mathrm{a}}$ & Reference & $2.14(1.10-4.17)$ & 0.0268 & $6.36(2.49-16.23)$ & 0.0004 \\
\hline Fully adjusted $\mathrm{OR}^{\mathrm{b}}$ & Reference & $1.90(0.91-3.96)$ & 0.0836 & $5.31(1.87-15.10)$ & 0.0029 \\
\hline \multicolumn{6}{|l|}{ ED visits } \\
\hline Rate (\%) & 16.8 & 36.2 & & 45.2 & \\
\hline Unadjusted OR & Reference & $2.80(1.66-4.72)$ & 0.0004 & 4.08 (I.83-9.09) & 0.0013 \\
\hline Adjusted $\mathrm{OR}^{\mathrm{a}}$ & Reference & $3.27(1.76-6.06)$ & 0.0005 & $5.71(2.18-14.92)$ & 0.0009 \\
\hline Fully adjusted $\mathrm{OR}^{\mathrm{b}}$ & Reference & $2.64(1.35-5.17)$ & 0.0063 & $4.05(1.31-12.49)$ & 0.0170 \\
\hline
\end{tabular}

Notes: ${ }^{a}$ Adjusted for age, sex, education, and marital status. ${ }^{b}$ Adjusted for age, sex, education, marital status, duration of diabetes, use of insulin, falls, ADL disability, and IADL disability.

Abbreviations: ADL, activities of daily living; IADL, instrumental activities of daily living; ED, emergency department.

odds of hospital admission and emergency department visits compared to being robust.

Our finding that being frail or pre-frail is common in older adults with diabetes is consistent with previous studies..$^{10,11}$ The major strength of our study is that we investigated the prevalence of frailty in a population-based national sample of older adults with diabetes. We found that $9.4 \%$ and $35.3 \%$ of Taiwanese older adults aged 65 years and above with diabetes were frail and pre-frail, respectively. One pilot study on a sample of 146 inpatients aged 60 years and older with type 2 diabetes in China also used the FRAIL scale and found a prevalence of frailty of $15.1 \%$ and pre-frailty of $37.7 \% .{ }^{11}$ Another similar small study of a sample of 198 outpatients aged 50 years and above with diabetes in the USA found a prevalence of frailty of $28.8 \%$ and pre-frailty of $38.9 \%$ also using the FRAIL scale. ${ }^{10}$

Our data showed that participants with diabetes who were frail were more likely to be older, have a longer duration of diabetes, be using insulin, have fallen during the past year, and report ADL and IADL disability (Table 1). Most previous research agrees that these attributes are important risk factors for hospitalization and emergency department visits among people with diabetes. ${ }^{16-18}$ Our results extend past research by demonstrating that the OR for hospital admission and emergency department visits in frail compared to robust participants did not greatly alter after adjustment for other factors (Table 2). This suggests that frailty itself is independently associated with hospitalization and emergency department visits among older adults with diabetes.

Our study has some limitations, and the results should be interpreted with caution. First, we did not have data on glycated hemoglobin, severity of diabetes, and diabetes complications. These factors may be associated with both health care use and frailty and may have confounded our results. Second, blood pressure and cognitive function are important prognostic factors in frail older adults with diabetes. However, in our study, only self-reported hypertension (confirmed by a medical professional) was available as we did not measure blood pressure. Information regarding selfreported dementia (confirmed by a medical profession) was available in our study and 29 of our participants had selfreported dementia, of whom 7 were frail and 20 were prefrail. However, as presence of dementia would likely impact on a participant's ability to complete our survey, it is possible that participants with dementia were under-represented in our study. Therefore, we may have underestimated the prevalence of cognitive impairment in older adults with diabetes. Third, due to the cross-sectional study design, causal relationships between frailty and health care use cannot be established. There could be a bidirectional connection between frailty and health care use in this population. Many inpatients do not return to their premorbid level of function at discharge and acquire new geriatric syndromes during hospitalization, leading to further functional decline and the development of frailty after hospitalization. ${ }^{19}$ However, prospective studies have also found that being frail is associated with a greater likelihood of hospitalization in older people with diabetes. ${ }^{10,11}$ Our findings could also reflect poorer health status that is linked to both frailty and increased use of health services.

Previous studies have found that diabetes-specific attributes, including hypoglycemia, hyperglycemia, and diabetes complications, are associated with hospitalization and emergency department visits. ${ }^{20-24}$ These attributes are also associated with frailty in older adults with diabetes. In a review of the incidence and the consequences of hypoglycemia, Abdelhafiz et al found that hypoglycemia 
was less recognized in older adults with diabetes and was also associated with reduced physical and cognitive function leading to frailty. ${ }^{20}$ In an analysis of data from the Adult Changes in Thought study, Zaslavsjy et al found a U-shape association between glucose levels and frailty in adults aged 65 years and older with diabetes. ${ }^{22}$ Prior research also suggests an association between hyperglycemia and frailty among older women with diabetes. ${ }^{23} \mathrm{Li}$ et al conducted a pilot study using the FRAIL scale to predict outcomes in older adults with type 2 diabetes and found that frail status was associated with diabetic nephropathy..$^{10}$ These observations provide a possible explanation for our finding of an association between frailty and increased odds of hospitalization and emergency department visits among older adults with diabetes.

We consider our findings to have important practical implications. The major strengths of using the FRAIL scale are that FRAIL includes biological factors, functional deficits, and accumulated illness, and is relatively simple and easy to use. Our results show that the FRAIL scale can identify older adults with diabetes who have higher odds of hospitalization and emergency department visits in the absence of laboratory and clinical indicators and in a relatively inexpensive manner. Our data further suggest that health care professionals should particularly target interventions aimed at improving frailty status toward older adults with diabetes who are older, less educated, unmarried, live alone, have longer duration of diabetes, use insulin, have fallen during the past year, and report ADL and IADL disability (Table 1). We also hope our findings will stimulate further efforts to design and implement specific interventions aimed at preventing frailty in patients with diabetes after hospitalization or emergency department visits. Further investigation is needed to explore the underlying causes of hospitalization and emergency department visits in older adults with diabetes with frailty and whether such individuals could benefit from interventions to reduce health care use.

\section{Conclusion}

In summary, our results contribute to the literature by providing new data on the prevalence of frailty as defined by the FRAIL scale among a population-based sample of community-dwelling older adults with diabetes. Our results highlight the high prevalence of frailty in community-dwelling older adults with diabetes. Moreover, among older adults with diabetes, being frail was associated with higher odds of hospitalization and emergency department visits during the past year, after taking into account other risk factors.

\section{Acknowledgments}

This study is based (in part) on data from the National Health Interview Survey Original Database provided by the Health Promotion Administration, Ministry of Health and Welfare, and the National Health Research Institutes. The interpretation and conclusions contained herein do not represent those of the Health Promotion Administration, Ministry of Health and Welfare, and the National Health Research Institutes. This study was supported (in part) by grants (MOST106-2410-H-182-019-MY2) from the Ministry of Science and Technology, Taiwan.

\section{Disclosure}

The authors report no conflicts of interest in this work.

\section{References}

1. Chen LK, Chen YM, Lin MH, Peng LN, Hwang SJ. Care of elderly patients with diabetes mellitus: a focus on frailty. Ageing Res Rev. 2010; 9(Suppl 1):S18-S22.

2. Morley JE, Abbatecola AM, Woo J. Management of comorbidities in older persons with type 2 diabetes. J Am Med Dir Assoc. 2017;18(8): 639-645.

3. Ricci NA, Pessoa GS, Ferriolli E, Dias RC, Perracini MR. Frailty and cardiovascular risk in community-dwelling elderly: a population-based study. Clin Interv Aging. 2014;9:1677-1685.

4. Chhetri JK, Zheng Z, Xu X, Ma C, Chan P. The prevalence and incidence of frailty in pre-diabetic and diabetic community-dwelling older population: results from Beijing longitudinal study of aging II (BLSA-II). BMC Geriatr. 2017;17(1):17-47.

5. Castrejón-Pérez RC, Gutiérrez-Robledo LM, Cesari M, PérezZepeda MU, Mellitus D. Diabetes mellitus, hypertension and frailty: a population-based, cross-sectional study of Mexican older adults. Geriatr Gerontol Int. 2017;17(6):925-930.

6. Fried LP, Tangen CM, Walston J, et al. Frailty in older adults: evidence for a phenotype. J Gerontol A Biol Sci Med Sci. 2001;56(3):M146-M157.

7. Cacciatore F, Testa G, Galizia G, et al. Clinical frailty and long-term mortality in elderly subjects with diabetes. Acta Diabetol. 2013;50(2): 251-260.

8. Chode S, Malmstrom TK, Miller DK, Morley JE, Frailty MJE. Frailty, diabetes, and mortality in middle-aged African Americans. J Nutr Health Aging. 2016;20(8):854-859.

9. Chang HY, Hsu CC, Pan WH, et al. Gender differences in trends in diabetes prevalence from 1993 to 2008 in Taiwan. Diabetes Res Clin Pract. 2010;90(3):358-364.

10. Liccini A, Malmstrom TK. Frailty and sarcopenia as predictors of adverse health outcomes in persons with diabetes mellitus. J Am Med Dir Assoc. 2016;17(9):846-851.

11. Li Y, Zou Y, Wang S, et al. A pilot study of the FRAIL scale on predicting outcomes in Chinese elderly people with type 2 diabetes. J Am Med Dir Assoc. 2015;16(8):714.e7-714.e12.

12. Shih YH, Chang HY, Lu MI, Hurng BS. Time trend of prevalence of self-reported cataract and its association with prolonged sitting in Taiwan from 2001 and 2013. BMC Ophthalmol. 2014;14:128.

13. Abellan van Kan G, Rolland Y, Bergman H, Morley JE, Kritchevsky SB, Vellas B. The I.A.N.A Task Force on frailty assessment of older people in clinical practice. J Nutr Health Aging. 2008;12(1):29-37.

14. Abellan van Kan G, Rolland YM, Morley JE, Vellas B. Frailty: toward a clinical definition. J Am Med Dir Assoc. 2008;9(2):71-72.

15. Woo J, Leung J, Morley JE. Comparison of frailty indicators based on clinical phenotype and the multiple deficit approach in predicting mortality and physical limitation. J Am Geriatr Soc. 2012;60(8):1478-1486. 
16. Geller AI, Shehab N, Lovegrove MC, et al. National estimates of insulinrelated hypoglycemia and errors leading to emergency department visits and hospitalizations. JAMA Intern Med. 2014;174(5):678-686.

17. Ustulin M, Woo J, Woo JT, Rhee SY. Characteristics of frequent emergency department users with type 2 diabetes mellitus in Korea. J Diabetes Investig. 2018;9(2):430-437.

18. Moss SE, Klein R, Klein BE. Risk factors for hospitalization in people with diabetes. Arch Intern Med. 1999;159(17):2053-2057.

19. Lakhan P, Jones M, Wilson A, Courtney M, Hirdes J, Gray LC. A prospective cohort study of geriatric syndromes among older medical patients admitted to acute care hospitals. J Am Geriatr Soc. 2011;59(11):2001-2008.

20. Abdelhafiz AH, Rodríguez-Mañas L, Morley JE, Sinclair AJ. Hypoglycemia in older people - a less well recognized risk factor for frailty. Aging Dis. 2015;6(2):156-167.
21. Alexiu CJ, Chuck A, Jelinski SE, Rowe BH. Presentations for hypoglycemia associated with diabetes mellitus to emergency departments in a Canadian province: a database and epidemiological analysis. Diabetes Res Clin Pract. 2017;130(8):229-236.

22. Zaslavsky O, Walker RL, Crane PK, Gray SL, Larson EB. Glucose levels and risk of frailty. J Gerontol A Biol Sci Med Sci. 2016;71(9): 1223-1229.

23. Blaum CS, Xue QL, Tian J, Semba RD, Fried LP, Walston J. Is hyperglycemia associated with frailty status in older women? $\mathrm{J} \mathrm{Am}$ Geriatr Soc. 2009;57(5):840-847.

24. Clarke PM, Glasziou P, Patel A, et al. Event rates, hospital utilization, and costs associated with major complications of diabetes: a multicountry comparative analysis. PLoS Med. 2010;7(2):e1000236.
Clinical Interventions in Aging

\section{Publish your work in this journal}

Clinical Interventions in Aging is an international, peer-reviewed journal focusing on evidence-based reports on the value or lack thereof of treatments intended to prevent or delay the onset of maladaptive correlates of aging in human beings. This journal is indexed on PubMed Central, MedLine,

\section{Dovepress}

CAS, Scopus and the Elsevier Bibliographic databases. The manuscript management system is completely online and includes a very quick and fair peer-review system, which is all easy to use. Visit http://www.dovepress. com/testimonials.php to read real quotes from published authors. 